\title{
Can adverse effects of dietary fat intake be overestimated as a consequence of dietary fat underreporting?
}

\author{
Berit Lilienthal Heitmann ${ }^{1,2, *}$ and Lauren Lissner ${ }^{3}$ \\ ${ }^{1}$ Research Unit for Dietary Studies and Danish Epidemiology Science Centre, Institute of Preventive Medicine, \\ Copenhagen University Hospital, DK-1399 Copenhagen K, Denmark: ${ }^{2}$ Research Centre for Prevention and Health, \\ Glostrup University Hospital, Glostrup, Denmark: ${ }^{3}$ Department of Internal Medicine, Sahlgrenska University Hospital, \\ Gothenborg University, Gothenborg, Sweden
}

\section{Submitted 29 January 2004: Accepted 3 February 2005}

\begin{abstract}
Objective: To describe the consequences of systematic reporting bias by the obese for diet-disease relationships.

Design: The present report used 24-hour urinary nitrogen and estimates of 24-hour energy expenditure to assess error in diet reporting, and examined the consequence of accounting for this error for associations between dietary fat intake and serum lowdensity lipoprotein (LDL)-cholesterol.

Setting: Sub-study to the Danish MONICA (Monitoring Trends and Determinants in Cardiovascular Disease) project, carried out in 1987-1988.

Subjects: A random sub-sample of the adult Danish male population $(n=152)$.

Results: Correcting dietary fat for underreporting error weakened, rather than strengthened, the association between dietary fat intake and LDL-cholesterol by reducing the slope of the regression from $\beta=3.4, P=0.02$ to $\beta=2.7, P=0.04$.

Conclusion: This example illustrates that systematic underreporting of dietary fat by high-risk groups such as the obese may produce an overestimated association. These results imply that previous epidemiological studies showing a positive association between percentage of energy from fat and other health outcomes, e.g. cancer and heart disease, may have overestimated the negative effects of a high-fat diet. If we were able to correctly assess dietary fat intake in general populations, recommendations for fat intake may be more liberal than the 30\% suggested today. Improved assessment of fat intake in epidemiological studies is necessary for future development of evidence-based recommendations for diet and health .
\end{abstract}

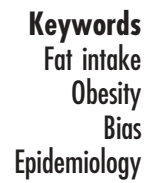

For more than two decades, authorities have recommended that dietary fat intake be reduced to $30-35 \%$ of total energy ${ }^{1,2}$. These recommendations are based on results from epidemiological, clinical and animal studies, suggesting that a reduction in dietary fat intake is associated with improvement in obesity, dyslipidaemia and hypertension, which are known risk factors for heart disease, a number of cancers, diabetes and gallstones. Indeed, it has been suggested that $35 \%$ of all cancer deaths $^{3}$, in particular cancers of the breast, colon and prostate, may be attributable to diet. It is likely that a similar proportion of coronary deaths are related to dietary factors.

Weak associations between dietary fat and cancer or cardiovascular disease mortality

Whereas most evidence from clinical and animal experiments would suggest that a reduction in fat intake is associated with improved lipids and insulin resistance, the evidence from observational studies is somewhat weaker, and more inconsistent. It is often assumed that the weaker positive associations found in observational studies are partly dependent on poor-quality information on diet, and that the true risk associated with a high dietary fat intake is underestimated ${ }^{4}$.

\section{Dietary underreporting}

For public health purposes, a valid measure of diet and disease associations is important as such associations are used to calculate relative and attributable risk fractions. However, the temporal sequence underlying the relationships between, for instance, dietary intake and cancer development can lead to errors in dietdisease relationships, since reported diet 30 years ago may not reflect subsequent intake. In general, random misclassification of a risk factor, such as dietary fat, leads to an underestimation of diet-related health effects $^{5,6}$. This depends on the fact that correction for random measurement error usually increases the slope of a diet-disease relationship, because the correction 
reduces the variability of the independent variable (the health outcome). However, it is a misconception that random errors in dietary intake data always result in an underestimation of the true health risk $^{4,7}$. Furthermore, the fact that differential misclassification can bias associations profoundly, i.e. different error in subjects with or without disease, is often neglected in population-based studies. For instance, it has been shown repeatedly that diet underreporting is more pronounced in certain subgroups of the population (e.g. high energy consumers, obese, complying personalities, etc. $)^{4,8,9}$. Indeed, obese subjects have been found to underreport up to half of their total energy intake $^{8,9}$. Moreover, a recent report suggests that obese individuals in particular underreport intake of fat and/or carbohydrate $^{10}$. The present paper is based on the hypothesis that the health risk attributed to high fat intake may be overestimated, if this specific underreporting in fat intake in the obese is not considered in studies showing a positive association between fat intake and a health outcome. Or, simply stated, adverse effects of a high fat intake may be exaggerated because, when correcting for systematic measurement error, the underreporters in the middle of the distribution will be moved to the upper end, attenuating the regression slope. In this context, the opportunity has been taken to recalculate data on associations between fat intake and low-density lipoprotein (LDL)-cholesterol to demonstrate, in an observational setting, the effect of diet-reporting bias of fat intake on serum LDLcholesterol levels. The association between dietary fat intake and serum LDL-cholesterol was chosen as an example for several reasons. First, because a high intake of saturated fatty acids is known to raise LDLcholesterol, second because the Danish diet is generally high in saturated fat, and third because obesity is positively associated with both high LDL-cholesterol levels and dietary fat. Thus, misclassification of fat exposure is not random.

\section{Subjects and method}

\section{Subjects}

The study was part of the Danish MONICA (Monitoring Trends and Determinants in Cardiovascular Disease) project, carried out between 1987 and 1988 ${ }^{10}$. (The MONICA studies are international studies conducted under the auspices of the World Health Organization (WHO) to monitor trends in, and determinants of, mortality from cardiovascular disease.) The study population included 276 Danish men aged 35, 45, 55 and 65 years, who were selected, at random, from a larger population sample of 2290 subjects $^{10}$. Of the 276 contacted, 235 attended the health examination including measurement of LDL-cholesterol and body composition by impedance. Of these, 218 agreed to further partici- pation in the diet study. In all 152 subjects completed both the diet history interview and returned a complete 24-hour urine sample. This was the same group in whom underreporting bias was described earlier ${ }^{10}$. The project was approved by the Ethical Committee for Copenhagen County, and is in accordance with the Helsinki II Declaration.

\section{Diet}

One trained dietitian interviewed all subjects by the diet history method. Average daily intakes were calculated from responses describing the previous month. Meal patterns, dishes and food items were described using a pre-coded interview form. Quantities were assessed by the use of food models, series of photographs, cups and measures. Nutrient calculations were carried out with the DANKOST program, which is derived from the Danish food composition tables ${ }^{11}$.

\section{Urine collection}

All subjects were instructed, orally and in writing, in the collection of 24-hour urine. To monitor completeness, each participant was given three tablets containing $80 \mathrm{mg}$ $p$-aminobenzoic acid to be taken during the day of urine collection $^{12}$.

\section{4-Hour nitrogen excretion}

Protein intake was calculated from 24-hour nitrogen excretion according to the formula ${ }^{6}$ :

$\operatorname{Prot}_{\mathrm{u}}(\mathrm{g})=\left(\mathrm{N}_{\mathrm{u}}+2 \mathrm{~g}\right) \times 6.25$,

where $\operatorname{Prot}_{\mathrm{u}}$ is the calculated protein intake based on urine samples and $\mathrm{N}_{\mathrm{u}}$ is the nitrogen output in 24-hour urine measured in $\mathrm{g} \mathrm{day}^{-1}$.

\section{Analyses of urine}

Thirty microlitre urine samples were analysed for nitrogen by the flash combustion technique ${ }^{12}$ in an NA 1500 Nitrogen Analyser (Carlo Erba Strumentazione, Milan, Italy).

\section{Anthropometric data}

All anthropometric measurements were taken in accordance with WHO standards ${ }^{13}$. Height was measured to the nearest $0.5 \mathrm{~cm}$. Body weight was measured to the nearest $0.1 \mathrm{~kg}$ using a SECA scale, with subjects wearing only hospital underwear.

\section{Measurements of electrical impedance}

A BIA-103 RJL system analyser (RJL Systems, Detroit, MI, USA) was used to measure electrical impedance, following the instructions given by the manufacturer. The measurements were taken using tetrapolar electrode placement, with electrodes placed on the right hand and foot.

The algorithm used to estimate body fat (BF) from impedance was previously developed on a subgroup 
1324

belonging to the same sample of Danes ${ }^{14}$ :

$$
\begin{aligned}
\mathrm{BF}(\mathrm{kg})= & 0.819 \times \mathrm{BW}(\mathrm{kg})-0.279 \times \mathrm{Ht}^{2} / \mathrm{R}\left(\mathrm{cm}^{2} / \mathrm{ohm}\right) \\
& -0.064 \times \mathrm{sex} \times \mathrm{BW}(\mathrm{kg})+0.077 \times \text { age }(\text { years }) \\
& -0.231 \times \mathrm{Ht}(\mathrm{cm})+14.941,
\end{aligned}
$$

where BW is body weight, $\mathrm{Ht}$ is height and $\mathrm{R}$ is resistance. Sex is coded as 1 for men and 0 for women.

\section{Questionnaire data}

All participants answered questions about physical activity during leisure (mostly sitting; light activity at least 4 hours per week; active in sport at least 3 hours per week, or heavy work during leisure; active in competitive sport, several times per week) and physical activity during work (no work; mostly sitting; light activity, walks around; mostly walking and carrying; strenuous physical work).

\section{Basal energy expenditure}

Basal energy expenditure (BEE) was calculated according to the formula of Garby et $a l .^{15}$ :

$$
\mathrm{BEE}(\text { Joules })=116.76 \times \mathrm{FFM}+26.88 \times \mathrm{BF},
$$

where FFM is fat-free mass.

Average 24-hour energy expenditure (24EE) can be expressed as a multiple of BEE, known as the physical activity level (PAL). In men and women who were unemployed or whose occupational work was classified as sedentary, it is assumed that PAL is 1.55 and 1.56 , respectively. Factors of 1.78 and 1.64 were used when work was classified as light activity, whereas factors of 2.10 and 1.82 were used for heavy and strenuous work, or for people engaged actively in sports ${ }^{16}$.

\section{Measurement of LDL-cholesterol}

A blood sample was drawn after a $12 \mathrm{~h}$ overnight fast. LDLcholesterol concentration $\left(\mathrm{mmol}^{-1}\right)$ was calculated as the difference between total cholesterol and high-density lipoprotein-cholesterol, which was measured in the serum using commercial enzymatic methods (Boehringer Mannheim GmbH, Mannheim, Germany).

\section{Assessment of diet intake and diet underreporting}

Twenty-four-hour urinary nitrogen excretion is a wellrecognised biological marker of daily protein intake. By comparing protein intake from a diet history interview with protein intake estimated from the 24-hour nitrogen output, validated by administering $p$-aminobenzoic acid, and estimated energy expenditure, we recently demonstrated that obese individuals tend to underreport their total energy intake by up to $15 \%$ more than they underreport their energy intake from protein ${ }^{10}$ (Fig. 1). If energy from protein is proportionately overreported it follows that energy from the other macronutrients, e.g. fat and carbohydrate, must be underreported. We therefore
BL Heitmann and L Lissner

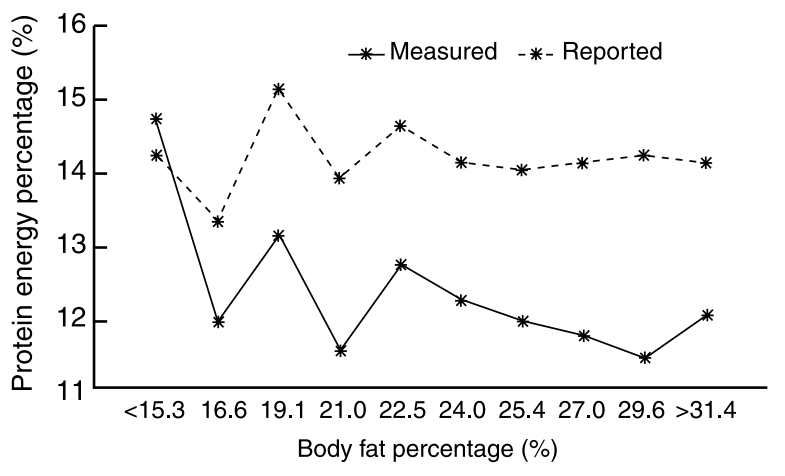

Fig. 1 Percentage of energy from protein, estimated from a diet history interview (Reported intake) and from urinary nitrogen and 24-hour energy expenditure (Measured intake), by percentage of body fat, adjusted for age and smoking, in 152 Danish men participating in the Danish MONICA (Monitoring Trends and Determinants in Cardiovascular Disease) project (modified from reference 10)

based our present study on the assumption that obese people tend to underreport fat- and/or carbohydrate-rich foods ${ }^{10}$. In this we used these data on measured energy and protein intakes to recalculate intake of energy from fat corrected for reporting bias. As a simplification, we assumed that the observed underreporting occurred in the fat fraction only. Hence, the corrected values presented here are calculated using individual data on reporting bias, assuming that the overreported energy from protein is compensated by underreporting of a similar amount of energy from fat, only. This assumption, of course, is likely to exaggerate reality, but is chosen simply as a matter of illustration.

\section{Statistical analyses}

Univariate and multiple linear regression analyses were used to describe the statistical associations between fat intake and LDL-cholesterol. We used both linear and logistic regression to describe the effect of correcting for underreporting, in order to demonstrate whether similar trends were evident both with and without assumptions about linearity.

\section{Results}

Non-participation has been described in detail earlier, and showed no differences in terms of age, body weight, height, body mass index (BMI), percentage body fat, prevalence of obesity (BMI $>30 \mathrm{kgm}^{-2}$ ) or LDLcholesterol level among the following three groups: (1) the subjects who undertook the diet history interview but gave an incomplete urine collection; (2) the subjects who never returned a urine collection; and (3) the subjects, included in the present study, who completed both (all $P>0.30)^{10}$.

Subject characteristics are given in Table 1. LDLcholesterol level, energy and fat intakes, age and all 
Table 1 Characteristics of the 152 men

\begin{tabular}{|c|c|}
\hline Age (years) & $49.5 \pm 10.9$ \\
\hline LDL-cholesterol (mmoll-1) & $4.1 \pm 1.1$ \\
\hline \multicolumn{2}{|l|}{ Anthropometric measures } \\
\hline Body weight $(\mathrm{kg})$ & $78.6 \pm 11.6$ \\
\hline Body height (m) & $1.76 \pm 0.07$ \\
\hline $\mathrm{BMI}\left(\mathrm{kg} \mathrm{m}^{-2}\right)$ & $25.3 \pm 3.2$ \\
\hline Body fat (kg) & $18.6 \pm 7.1$ \\
\hline Body fat (\%) & $23.1 \pm 6.0$ \\
\hline \multicolumn{2}{|l|}{ Dietary measures } \\
\hline Energy intake (MJ) & $10.2 \pm 2.6$ \\
\hline Predicted energy intake (MJ) & $13.5 \pm 2.3$ \\
\hline Fat intake $(\mathrm{g})$ & $108.2 \pm 29.8$ \\
\hline Energy from fat $(\%)$ & $40.6 \pm 5.9$ \\
\hline Adjusted energy from fat (\%) & $42.5 \pm 6.5$ \\
\hline \multicolumn{2}{|l|}{ Questionnaire data } \\
\hline No. (\%) of subjects physically active during work* & $32 / 152(21)$ \\
\hline No. (\%) of subjects taking part in sports $†$ & $33 / 152(22)$ \\
\hline No. (\%) of current smokers & $68 / 152(45)$ \\
\hline
\end{tabular}

LDL - low-density lipoprotein; BMI - body mass index. *Mostly walking and carrying orstrenuous physical work.

†Active in sport atleast 3 hours per week, or heavy work during leisure, or active in comptetitive sport several times per week.

anthropometric values, except height, varied with degree of obesity (data not shown).

The Danish diet is high in fat, and Fig. 2 displays the effect of correcting reported energy from fat for underreporting. Underreporting was seen, in particular, in men with the high fat intake. Univariately, strong positive associations were found between percentage energy from fat and LDL-cholesterol, both before and after correction for the reporting bias. Associations were stronger for the reported $(\beta=4.0 \pm 1.5, P=0.009)$ than for the corrected intakes $(\beta=3.5 \pm 1.3, P=0.01)$ as illustrated in Fig. 3, where it can be seen that the slope of the regression between fat intake and serum LDL-cholesterol was shifted

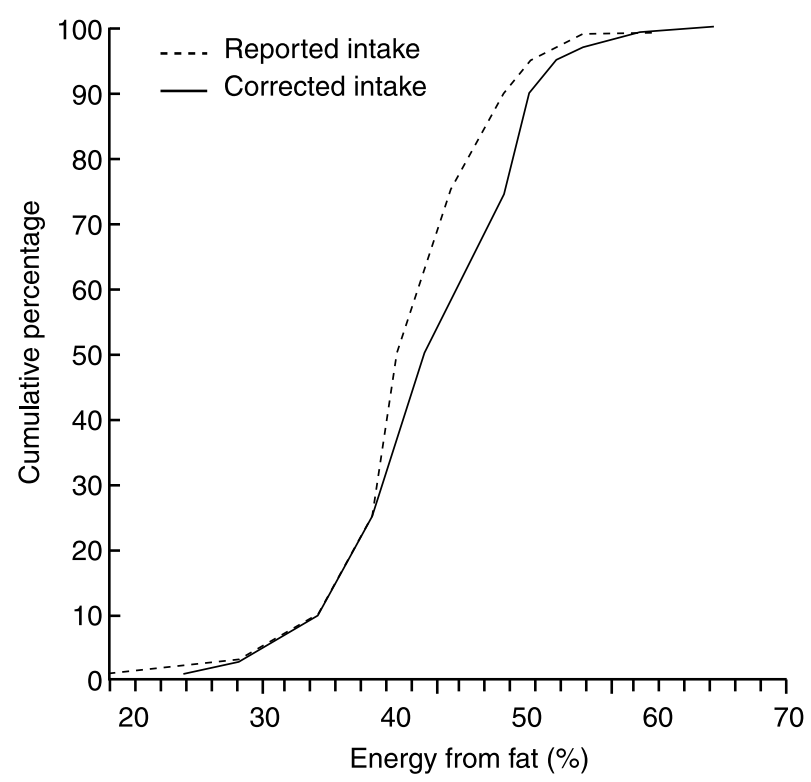

Fig. 2 Cumulative distribution curves showing percentage of energy from fat before (Reported intake) and after (Corrected intake) considering underreporting of fat intake

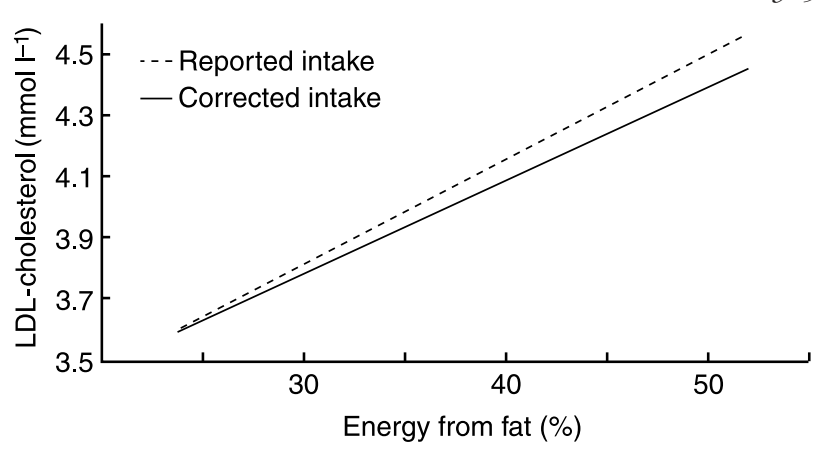

Fig. 3 Associations between low-density lipoprotein (LDL)-cholesterol level $\left(\mathrm{mmoll}^{-1}\right)$ and dietary fat intake (as a percentage of energy) before (Reported intake) and after (Corrected intake) considering underreporting of fat intake

downwards after correction. Due to the covariation between diet underreporting and obesity, adjustment for BMI in diet-disease relationships would be expected to compensate, in part, for the downward shift of the slope of the regression seen after correction for underreporting. However, although adjustment for BMI, as expected, weakened the associations, they still remained stronger between reported intake and LDL-cholesterol $(\beta=3.4 \pm 1.4, P=0.02)$ than between corrected intake and LDL-cholesterol $(\beta=2.7 \pm 1.3, P=0.04)$. Hence the correction for BMI did not explain the downwards shift of the slope after correction, potentially because underreporting was evident, even if weaker, also for the leaner subjects, as also indicated earlier ${ }^{10,17}$.

Indeed, since underreporting in the present study varies as a function of obesity, there is also underreporting far down in the distribution of body fatness. The dietary 'exposure' was redefined in ranked categories (tertiles) and LDL-cholesterol level was transformed to high versus normal (LDL-cholesterol $\left.>4.5 \mathrm{mmoll}^{-1}\right)^{18}$, based on which the odds ratios (ORs) were calculated. Comparison of the upper with the lower tertile of reported fat intake yielded $\mathrm{OR}=2.2(P=0.06)$, whereas comparison of the upper and the lower tertile for corrected fat intake reduced the OR to $1.4(P=0.31)$. Table 2 gives the OR by tertile of fat intake before and after correction for underreporting bias. Before correction a higher fat intake tended to be associated with a higher LDL-cholesterol level $(P$ for

Table 2 Odds ratio for high LDL-cholesterol by tertile of fat intake before and after correction for underreporting bias among 152 men

\begin{tabular}{|c|c|c|c|c|}
\hline Fat intake & First tertile* & $\begin{array}{l}\text { Second } \\
\text { tertile } \\
\text { (vs. first) }\end{array}$ & $\begin{array}{c}\text { Third tertile } \\
\text { (vs. first) }\end{array}$ & $\begin{array}{c}\text { Trend } \\
\text { estimate }(\beta)\end{array}$ \\
\hline Reported & 1.0 & 1.4 & 2.2 & 1.48 \\
\hline $95 \% \mathrm{Cl}$ & - & $0.6-3.2$ & $0.95-5.0$ & $0.98-2.25$ \\
\hline Corrected & 1.0 & 2.4 & 1.6 & 1.22 \\
\hline $95 \% \mathrm{Cl}$ & - & $1.0-5.5$ & $0.7-3.7$ & $0.81-1.86$ \\
\hline
\end{tabular}

LDL - low-density lipoprotein; $\mathrm{Cl}$ - confidence interval.

* Tertiles were calculated based on the specific distribution for reported and corrected fat intakes, respectively. 
trend $=0.06)$, whereas after correction this was no longer the case $(P$ for trend $=0.33$ ).

\section{Discussion}

The present study demonstrates that, in accordance with theoretical considerations, a selective underreporting of fat intake by obese individuals would overestimate the 'true' association between dietary fat intake and LDLcholesterol. These findings have implications for understanding the nature of the associations between diet and disease in general, suggesting that previous observational studies showing a positive association between percentage energy from fat and health outcomes are likely to have overestimated the true effects of the fat intake. In general, overreporting of energy from protein was not more than approximately $2 \%$ (14\% vs. $12 \%)$. However, this overreported protein must be accompanied by underreported energy from fat and/or carbohydrate. Although an error of $2 \%$ may seem small, it represents a considerable difference in eating habits. In this context, it has been estimated in a cross-sectional study on associations between oxidative fat energy and body fat that an average increase in dietary fat energy of only $1.6 \%$ may result in a $10 \mathrm{~kg}$ increase in body fat mass ${ }^{19}$. Others have estimated that each $1 \%$ reduction in total cholesterol concentration is associated with a reduction in cardiovascular disease of at least $2 \%{ }^{20}$. This illustrates first, that what might be seen as a minor misclassification error in fat energy intake may have major consequences for weight development and disease related to weight; and second, that the importance assigned to a high dietary fat intake in promoting heart disease, and possibly cancer too, may be exaggerated.

One limitation of this study is the assumption that the underreporting occurred in the fat fraction only, and that it would be an equally valid simplification to assume that it occurred only in the reporting of carbohydrate. In reality, underreporting is probably taking place for both fat- and carbohydrate-rich foods. The noted consequences are therefore likely to be less than those found in the present study. A second possible limitation of the results involves generalising of the results. It is generally found that the obese, in particular, underreport socially desirable foods ${ }^{10,21}$. It is possible with intensified public health campaigns and official recommendations to reduce fat intake; this type of bias will affect whole populations and not specifically the obese. Thus, it may not be possible to extrapolate these results to other contemporary epidemiological studies. Finally, it should be noted that the type of bias described here would be most likely in studies of dietary fat as a possible predictor of obesity-related risk factors and endpoints. For most studies in nutritional epidemiology, such associations are likely to be positive, and underreporting to result in an overestimated effect. However, in descriptive epidemiological studies identifying high fat consumers, the opposite bias could occur.
Hence, the predictive value of obesity-related variables (e.g. age) vis-à-vis fat intake could be underestimated by obesity-related underreporting. It should also be pointed out that this type of a bias could result in an underestimate of the proportion of high fat consumers who are obese, and vice versa.

In conclusion, differential biases in diet reporting can have unpredictable consequences when studied in relation to obesity and obesity-related disease, and our results indicate that it is not sufficient to control for obesity in order to correct this bias. Furthermore, the present study suggests that the risk of developing high LDL-cholesterol from a high fat consumption may be generally overestimated, and the same may be true for cancer and other cardiovascular disease endpoints. However, the results of the present study do not suggest that fat intake is unrelated to health; merely that the risk attributed to a high dietary fat intake may be overrated. Since our recommendations for fat intake are based on reported intakes, the present study would seem to indicate that, if we were able to correctly assess dietary fat intake in general populations, recommendations for dietary fat intake may be more liberal.

\section{References}

1 Committee on Diet, Nutrition, and Cancer, National Academy of Sciences. Diet, Nutrition, and Cancer. Washington, DC: National Academy Press, 1982.

2 Keyes A. Prevention of coronary heart disease. Official recommendations from Scandinavia. Circulation 1968; 38: 227-8.

3 Doll R, Peto R. The causes of cancer. Quantitative estimates of avoidable risks of cancer in the United States today. Journal of the National Cancer Institute 1981; 66 : $1191-308$.

4 Hebert JR, Clemow L, Pbert L, Ockene IS, Ockene J. Social desirability bias in dietary self-report may compromise the validity of dietary intake measures. International Journal of Epidemiology 1995; 24: 389-98.

5 Wu ML, Whittemore AS, Jung DL. Errors in reported dietary intakes. II. Long-term recall. American Journal of Epidemiology 1988; 128: 1137-45.

6 Willett W. An overview of issues related to the correction of non-differential exposure measurement error in epidemiologic studies. Statistics in Medicine 1989; 8: 1031-40.

7 Heitmann BL. Social desirability bias in dietary self-report may compromise the validity of dietary intake measures. Implications for diet-disease relationships. International Journal of Epidemiology 1996; 25: 222-3.

8 Heitmann BL. The influence of fatness, weight change, slimming history and other lifestyle variables on diet reporting in Danish men and women aged 35-65 years. International Journal of Obesity and Related Metabolic Disorders 1993; 17: 329-36.

9 Black AE, Goldberg GR, Jebb SA, Livingstone MBE, Cole TJ, Prentice AM. Critical evaluation of energy intake data using fundamental principles of energy physiology: 2. Evaluating the results of published surveys. European Journal of Clinical Nutrition 1991; 45: 583-99.

10 Heitmann BL, Lissner L. Dietary underreporting by obese individuals - is it specific or non-specific? British Medical Journal 1995; 311: 986-9. 
11 Møller A, ed. Levnedsmiddeltabeller 1985 [Danish Food Composition Tables 1985], 2nd ed. Ministry of the Environment Publication No. 75. Søborg: National Food Agency, 1986.

12 Bingham S, Cummings JH. The use of 4-aminobenzoic acid as a marker to validate the completeness of $24 \mathrm{~h}$ urine collections. Clinical Science 1983; 64: 629-35.

13 World Health Organization (WHO). Measuring Obesity Classification and Description of Anthropometric Data. Report on a WHO Consultation of the Epidemiology of Obesity, Warsaw, 21-23 October 1987. Nutrition Unit Document, EUR/ICP/NUT 125. Copenhagen: WHO, 1989.

14 Heitmann BL. Prediction of body water and fat in adult Danes from measurement of electrical impedance. A validation study. International Journal of Obesity 1990; 14: 789-802.

15 Garby L, Garrow JS, Jørgensen B, lammert O, Madsen K, Sørensen P. Relation between energy expenditure and body composition in man: specific energy expenditure in vivo of fat and fat-free tissue. European Journal of Clinical Nutrition 1988; 42: 301-5.

16 World Health Organization (WHO). Energy and Protein Requirements. Report of a Joint Food and Agriculture
Organization/WHO/United Nations University Expert Consultation. Technical Report Series No. 724. Geneva: WHO, 1985.

17 Jacobsen BK, Thelle DS. Risk factors for coronary heart disease and level of education. The Tromsö Heart Study. American Journal of Epidemiology 1988; 127: 923-32.

18 Prevention of coronary heart disease: scientific background and new clinical guidelines. Recommendations of the European Atherosclerosis Society prepared by the International Task Force for Prevention of Coronary Heart Disease. Nutrition, Metabolism, and Cardiovascular Diseases 1992; 2: 113-56.

19 Astrup A, Buemann B, Western P, Toubro S, Raben A, Christensen NJ. Obesity as an adaptation to a high fat diet: evidence from a cross-sectional study. American Journal of Clinical Nutrition 1994; 59: 350-5.

20 Tyroler H. Review of lipid-lowering trials in relation to observational epidemiologic studies. Circulation 1987; 76 : 515-22.

21 Lissner L, Heitmann BL, Lindroos AK. Measuring intake in free-living humans: a question of bias. Proceedings of the Nutrition Society 1998; 57: 333-9. 